\title{
On the Gravitational Instability of a Medium in Nonuniform Rotation
}

\author{
N. Bel and E. Schatzman \\ Institut d'Astrophysique, Paris, France
}

\section{INTRODUCTION}

$\mathrm{W}^{\mathrm{E}}$ E examine here the problem of the gravitational instability of a medium in nonuniform rotation. We seek therefore to resolve a problem differing somewhat from that examined by Chandrasekhar ${ }^{1}$ and Fricke. ${ }^{2}$ We imagine a gaseous medium in rotation in a potential field, $\phi$, and consider only the plane problem. In the unperturbed motion, the particles describe circular orbits in uniform motion. We examine the stability against perturbations.

\section{FORMULATION OF THE EQUATIONS}

Suppose that $\omega(r)$ is the angular velocity of rotation; $u(r), v(r)$ are the radial and tangential components of the perturbation velocity in the plane of symmetry of the system; $\delta \phi(r)$ is the potential of the perturbed gravitational field, and $\delta \rho(r)$ is the perturbation of the density. We suppose that the perturbation of the medium develops at constant temperature, that the density of the nonperturbed medium is uniform, and that the perturbation is independent of the angle of position.

We have the defining equations,

Dynamic:

$$
\begin{gathered}
\rho_{0}\left(\frac{\partial u}{\partial t}-2 v \omega\right)=\rho_{0} \frac{\partial \delta \varphi}{\partial r}-\frac{k T}{\mu} \frac{\partial \delta \rho}{\partial r} \\
\frac{\partial v}{\partial t}+u \frac{\partial}{\partial r} r \omega+u \omega=0 .
\end{gathered}
$$

Continuity:

$$
\frac{\partial}{\partial t} \delta \rho+\rho_{0} \frac{\partial u}{\partial r}+\rho_{0} \stackrel{u}{r}=0
$$

Poisson's equation:

$$
\frac{1}{r} \frac{\partial}{\partial r}(\underset{r}{\stackrel{\partial}{\partial r} \delta \phi})=-4 \pi G \delta \rho
$$

One seeks solutions of the form

$$
\begin{gathered}
\delta \rho=f(r) e^{i \sigma t}, \\
u=A(r) e^{i \sigma t}, \\
v=B(r) e^{i \sigma t} .
\end{gathered}
$$

${ }^{1}$ S. Chandrasekhar, Monthly Notices Roy. Astron. Soc. 113, 667; (1953); Astrophys. J. 119, 7 (1954)

2 W. Fricke, Astrophys. J. 120, 356 (1954).
If $\sigma$ has a negative imaginary part, the perturbation is unstable.

Poisson's equation gives, after integration

$$
\frac{\partial}{\partial r} \delta \varphi=-\frac{4 \pi G}{r} e^{i \sigma t} \int_{r 0}^{r} x f(x) d x
$$

Likewise, the equation of continuity gives

$$
A(r)=-\frac{i \sigma}{\rho_{0}}-\frac{1}{r} \int_{r_{0}}^{r} x f(x) d x .
$$

Finally, the equations of motion may be written

$$
i \sigma B(\boldsymbol{r})+2 \omega A(\boldsymbol{r})+A(\boldsymbol{r}) \boldsymbol{d \omega} \frac{d \omega}{d \boldsymbol{r}}=0,
$$

$\rho_{0}[i \sigma A(r)-2 B(r) \omega(r)]$

$$
=-\frac{4 \pi G \rho_{0}}{r} \int_{r 0}^{r} x f(x) d x-\frac{k T}{\mu} \frac{d f}{d r} .
$$

One may eliminate $A$ and $B$ between Eqs. (7)-(9) and obtain

$$
\begin{aligned}
& {\left[\sigma^{2}+4 \pi G \rho_{0}-2 \omega\left(2 \omega+r \frac{d \omega}{d r}\right)\right]} \\
& \quad \times \int_{r_{0}}^{r} x f(x) d x+\frac{k T}{\mu} r \frac{d f}{d r}=0 .
\end{aligned}
$$

If one introduces as a variable

$$
F(r)=\int_{r 0}^{r} x f(x) d x
$$

he obtains the differential equation

$$
F^{\prime \prime}-\frac{F^{\prime}}{r}+\frac{\mu}{k T}\left[\sigma^{2}+4 \pi G \rho_{0}-\frac{2 \omega}{r}\left(\omega r^{2}\right)^{\prime}\right] F=0 .
$$

In a region where the coefficient of $\mu / k T$ is essentially constant, this equation has a Bessel function for solution. Far from the center one is able as a first approximation to treat $r=r_{0}$ as a constant. One may set

$$
F \sim e^{\lambda r}
$$


and obtain

$$
\lambda=\frac{1}{2 r_{0}} \pm\left\{\frac{1}{4 r_{0}^{2}}-\frac{\mu}{k T}\left[\sigma^{2}+4 \pi G \rho_{0}-\frac{2 \omega}{r}\left(\omega r^{2}\right)^{\prime}\right]\right\}^{\frac{1}{2}} .
$$

When the radical is imaginary, $F$ is a sinusoidal function in the vicinity of $r=r_{0}$. This situation corresponds to the condition

$$
\sigma^{2}+4 \pi G \rho_{0}-\frac{2 \omega}{r}\left(\omega r^{2}\right)^{\prime}-\frac{c^{2}}{4 r_{0}^{2}}>0 .
$$

This condition is satisfied if

$$
4 \pi G \rho_{0}-\frac{2 \omega}{r}\left(\omega r^{2}\right)^{\prime}-\frac{c^{2}}{4 r_{0}^{2}}>0
$$

The half-period of an oscillation of density is given by

$$
\frac{\pi^{2}}{l^{2}}=\frac{1}{c^{2}}\left[\sigma^{2}+4 \pi G \rho_{0}-\frac{2 \omega}{r}\left(\omega r^{2}\right)^{\prime}-\frac{c^{2}}{4 r_{0}^{2}}\right] .
$$

There is instability if

$$
\frac{\pi^{2} c^{2}}{l^{2}}-4 \pi G \rho_{0}+\frac{2 \omega}{r}\left(\omega r^{2}\right)^{\prime}+\frac{c^{2}}{4 r_{0}^{2}}<0 .
$$

If $\omega=0, r_{0}=\infty$, one recovers Jean's instability criterion. But one notes the appearance of a new condition. In effect, the condition (18) is not satisfied for a critical wavelength if the condition (16) is satisfied.

\section{DISCUSSION}

In terms of the velocity $V(\boldsymbol{r})$ for circular motion the condition (16) may be written

$$
4 \pi G \rho_{\mathrm{gas}}>\frac{c^{2}}{4 r^{2}}+\frac{2 V}{r}\left(\frac{V}{r}+\frac{d V}{d r}\right) .
$$

Near the center of the galaxy,

$$
4 \pi G \rho_{\text {gas }}>\frac{c^{2}}{4 r^{2}}+\frac{16 \pi}{3} G \rho_{\text {total }} .
$$

In the exterior regions

$$
4 \pi G \rho_{\text {gas }}>\frac{c^{2}}{4 r^{2}}+\frac{4 \pi}{3} G \rho_{\text {central }} .
$$

In all cases, the term in $c^{2} / r^{2}$ is negligible. In the central regions, the condition (20) is not satisfied, since it leads to

$$
\rho_{\text {gas }}>\frac{4}{3} \rho_{\text {total }},
$$

which is absurd. In the peripheral regions

$$
\rho_{\text {gas }}>\frac{1}{3} \rho_{\text {central }} \text {. }
$$

With the constants of the differential rotation, we obtain in the solar neighborhood

$$
\rho_{\text {gas }}>1.10^{-24} \mathrm{~g} \mathrm{~cm}^{-3} \text {. }
$$

This supplementary condition is possibly capable of explaining the presence or the absence of spiral arms in the galaxies, according to whether it is or is not satisfied in these regions of space.

\section{DISCUSSION}

E. A. SPIEGEL, University of Michigan Observatory, Ann Arbor, Michigan: At what distance from the galactic center would the instability appear in our own galaxy?

E. Schatzman, Institut d'Astrophysique, Paris, France: I have not calculated it because I do not know the gas density very close to the center, but I would estimate 1000 to $2000 \mathrm{pc}$ from the center.

E. A. SPIEgEL : The figure one or two kiloparsecs was also, I believe, quoted by de Vaucouleurs as the radius of the rings in the many-armed galaxies.

D. L. LAYZER, Harvard College Observatory, Cambridge, Massachusetts: I think I am right that you have not taken into account the vertical extension in this case. I think if you do that you will find that the radius for gravitational instability is of the same order of magnitude as the vertical extension. I think quite generally, in any system finite or infinite, the radius for gravitational instability is always of the same order of magnitude as a typical dimension of the system. So, in the case of an infinite system such as you considered here, if you take into account the gravitational field in the quiescent medium-that is, if you do the zeroorder approximation properly-you will find that the radius for gravitational instability is infinite.

E. SCHATZMAN : I agree in some respects with what you say, but the point is this: you might have vertical condensation of about the thickness of the system. But that does not mean that you would have condensation in the plane of symmetry of the system. That calculation means only to limit the possibility of condensation in the plane of symmetry of the system. 